\title{
Algoritmo para el manejo de la aspiración pulmonar crónica en pediatría
} Algorithm for the management of chronic pulmonary aspiration in pediatrics

\author{
Dra. Giselle Cuestas ${ }^{a}$ Dra. Verónica Rodrígueza ${ }^{a}$ Dr. Patricio Bellia Munzón $n^{a}$ y Dr. Gastón Bellia Munzón ${ }^{b}$
}

\section{RESUMEN}

La aspiración pulmonar es el pasaje de alimentos, reflujo gastroesofágico y / o saliva a la vía aérea de manera suficiente que ocasione síntomas respiratorios crónicos o recurrentes. Es una causa importante de neumonía recurrente, enfermedad pulmonar progresiva, bronquiectasias e, incluso, muerte.

La aspiración es intermitente y, con frecuencia, ocurre en niños con anomalías médicas subyacentes o síndromes que ocasionan síntomas respiratorios similares a la aspiración pulmonar crónica, por lo que, muchas veces, el diagnóstico de aspiración se demora hasta que haya una lesión pulmonar significativa. Se describen los métodos diagnósticos disponibles y sus limitaciones, y las opciones de tratamiento de la aspiración pulmonar crónica en la población pediátrica.

Palabras clave: aspiración respiratoria, disfagia, videofluoroscopía, trastornos de deglución, pediatría.

\begin{abstract}
Pulmonary aspiration is the passage of food, gastroesophageal reflux and/or saliva to the airway in a manner sufficient to cause chronic or recurrent respiratory symptoms. It is an important cause of recurrent pneumonia, progressive lung disease, bronchiectasis and even death.

Aspiration is intermittent and often occurs in children with underlying medical conditions or syndromes that cause respiratory symptoms similar to chronic pulmonary aspiration, so diagnosis of aspiration is often delayed until there is a significant lung injury.

The available diagnostic methods and their limitations, and treatment options of chronic pulmonary aspiration in the pediatric population are described.

Key words: respiratory aspiration, dysphagia, videofluoroscopy, deglutition disorders, pediatrics.
\end{abstract}

http: / / dx.doi.org/10.5546/ aap.2019.412

Cómo citar: Cuestas G, Rodríguez V, Bellia Munzón P, Bellia Munzón G. Algoritmo para el manejo de la aspiración pulmonar crónica en pediatría. Arch Argent Pediatr 2019;117(6):412-420.

a. Sección de Endoscopía Respiratoria, División de Otorrinolaringología.

b. Servicio de Cirugía.

Hospital General de Niños "Dr. Pedro de Elizalde",

Ciudad Autónoma de Buenos Aires, Argentina.

Correspondencia:

Dra. Giselle Cuestas: giselle_cuestas@yahoo.com.ar

Financiamiento: Ninguno.

Conflicto de intereses: Ninguno que declarar.

Recibido: 23-3-2019

Aceptado: 16-7-2019

\section{INTRODUCCIÓN}

La etiología de la aspiración pulmonar crónica (APC) es variable; puede ser estructural o funcional $\mathrm{y}$, frecuentemente, multifactorial, con anomalías neurológicas, inflamatorias y/o anatómicas que contribuyen a la aspiración.

Se debe sospechar la aspiración en los niños con tos o ahogo durante la alimentación, síntomas respiratorios persistentes o recurrentes (neumonía, sibilancias, tos crónica), sialorrea, retraso del crecimiento, tiempo de alimentación prolongado (mayor de 30 minutos), fonación húmeda, respiración ruidosa, apneas y cianosis con la deglución y / o signos radiológicos de lesión pulmonar crónica. $^{1-5}$

Aproximadamente, el $25 \%$ de la población pediátrica experimenta algún tipo de dificultad en la deglución (o disfagia), y la incidencia es mayor en los recién nacidos prematuros y en los infantes con ciertas enfermedades o anomalías, las cuales se resumen en la Tabla 1.1-3,6-9

Las consecuencias de la disfagia pueden ser graves al disminuir la eficacia y/o la seguridad de la deglución, y ocasionan desnutrición y deshidratación en el primer caso y obstrucción de la vía aérea o aspiración pulmonar en el segundo. ${ }^{4} \mathrm{Si}$ bien la aspiración es más frecuente en los niños con trastornos neurológicos (del $35 \%$ al $80 \%$ ), una proporción significativa de niños sin anomalías neurológicas presentan aspiración, por lo general, debido a anomalías anatómicas o inflamatorias. ${ }^{1,2,8,10,11}$

No hay una prueba de referencia (gold standard) para el diagnóstico de la APC, por lo que constituye un desafío. El enfoque de un equipo multidisciplinario permite la evaluación global coordinada y la planificación de la terapia. ${ }^{12}$ En los algoritmos propuestos, se mencionan los principales estudios complementarios para ayudar al pediatra en el diagnóstico certero y en el tratamiento adecuado (Figuras 1 y 2).

\section{FISIOPATOLOGÍA}

La APC puede ocurrir como resultado de la disfunción de deglución, del reflujo gastroesofágico (RGE), de la incapacidad 
de proteger adecuadamente la vía aérea de las secreciones orales y/o de las anomalías anatómicas que comunican la vía aérea con el esófago. ${ }^{5}$

Tabla 1. Causas de disfagia en pediatría

\begin{tabular}{|c|c|}
\hline \multirow[t]{3}{*}{$\begin{array}{l}\text { Origen } \\
\text { anatómico }\end{array}$} & $\begin{array}{l}\text { Malformaciones cráneo-faciales: } \\
\text { Síndromes genéticos } \\
\text { Fisura palatina } \\
\text { Macroglosia }\end{array}$ \\
\hline & $\begin{array}{l}\text { Anomalías de la vía aérea: } \\
\text { Hendidura laríngea } \\
\text { Parálisis cordal }\end{array}$ \\
\hline & $\begin{array}{l}\text { Anomalías cardiovasculares: } \\
\text { Anillos vasculares }\end{array}$ \\
\hline \multirow[t]{2}{*}{$\begin{array}{l}\text { Origen } \\
\text { neurológico }\end{array}$} & $\begin{array}{l}\text { Enfermedades neuromusculares: } \\
\text { Parálisis cerebral } \\
\text { Miastenia gravis }\end{array}$ \\
\hline & $\begin{array}{l}\text { Lesiones cerebrales adquiridas: } \\
\text { Traumatismo } \\
\text { Neoplasia } \\
\text { Infección }\end{array}$ \\
\hline \multirow[t]{3}{*}{ Otros } & $\begin{array}{l}\text { Enfermedades gastrointestinales: } \\
\text { Reflujo gastroesofágico } \\
\text { Esofagitis eosinofílica } \\
\text { Acalasia }\end{array}$ \\
\hline & $\begin{array}{l}\text { Lesiones por ingesta de cáusticos o } \\
\text { cuerpos extraños }\end{array}$ \\
\hline & $\begin{array}{l}\text { Antecedente de intubación orotraqueal } \\
\text { prolongada }\end{array}$ \\
\hline
\end{tabular}

La deglución es un proceso complejo que requiere la coordinación de acciones voluntarias e involuntarias. Después de la preparación oral, el bolo alimentario es enviado voluntariamente a la faringe (fase oral). Esto desencadena la fase faríngea involuntaria, en la cual el paladar blando cierra la nasofaringe; la laringe se eleva y se inclina anteriormente; la epiglotis se retroflexiona; las cuerdas vocales verdaderas y falsas se cierran, y los constrictores faríngeos se contraen secuencialmente para impulsar el bolo hacia el esófago. El esfínter esofágico superior se relaja de manera simultánea y se abre, lo que permite el paso del alimento, y el peristaltismo esofágico transporta el bolo hacia el estómago (fase esofágica). ${ }^{5}$ La alteración de la efectividad, la duración o la sincronización de alguno de estos componentes resulta en aspiración.

Se ha demostrado la asociación entre RGE y síntomas respiratorios, ya sea porque la exposición de la mucosa de la laringe al ácido disminuye la sensibilidad laríngea y aumenta el riesgo de aspiración o porque la estimulación de reflejos esófago-gástricos puede causar broncoespasmos sin penetración del contenido estomacal en la vía aérea, lo que hace difícil determinar si un niño con RGE y síntomas

FiguRA 1. Algoritmo para el manejo de la aspiración pulmonar crónica en el niño sin enfermedad neurológica ni muscular

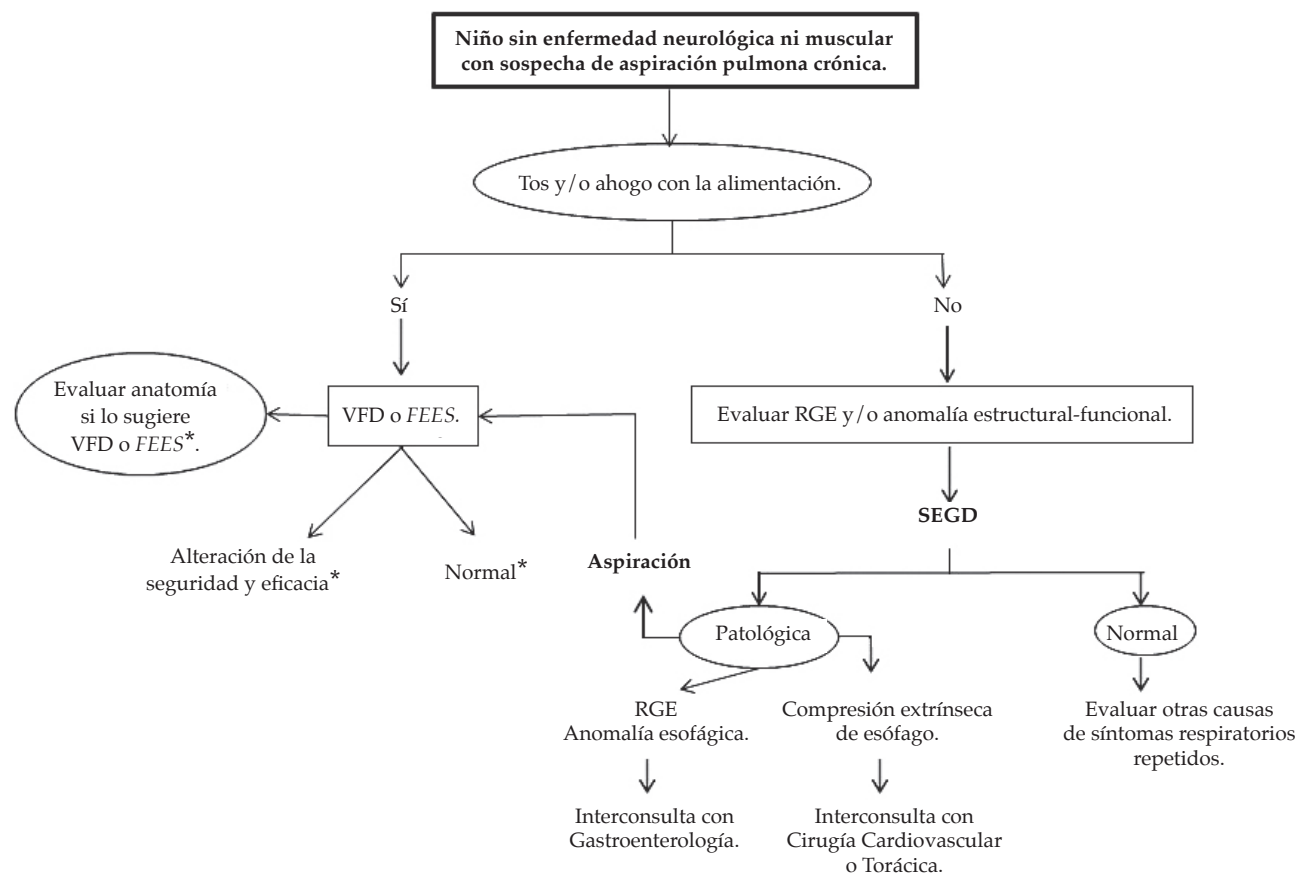

FEES: evaluación endoscópica flexible de la deglución; RGE: reflujo gastroesofágico; SEGD: seriada esofagogastroduodenal; VFD: videofluoroscopía de la deglución.

* Continúa en algoritmo de Figura 2. 
respiratorios crónicos se aspira o no. ${ }^{5}$ Los niños con RGE y síntomas respiratorios tendrían más episodios de reflujo no ácido que aquellos con RGE y síntomas gastrointestinales. ${ }^{13}$

La aspiración de saliva en los niños con deterioro neurológico se debe a la descoordinación grave de la deglución y a la ausencia de sensibilidad laríngea, más que al exceso de producción de saliva. También la aspiración de saliva es frecuente en los niños con sialorrea significativa, hendidura laríngea, parálisis cordal y síndromes, como el de coloboma, atresia/ estenosis coanal, disfunción de los nervios craneales y anomalías del oído (coloboma, heart defects, atresia choanae, growth retardation, genital abnormalities, and ear abnormalities; CHARGE, por sus siglas en inglés), Moebius, West y Pfeiffer. ${ }^{5}$ Entre las anomalías anatómicas que comunican la vía aérea con el esófago y ocasionan la aspiración, se deben considerar la hendidura laríngea y la fístula traqueoesofágica. ${ }^{5}$

\section{DIAGNÓSTICO}

El diagnóstico de APC requiere un alto índice de sospecha clínica, y su confirmación se hace por exámenes complementarios que evalúan las consecuencias (evidencia radiográfica de aspiración) y/o identifican las causas y los factores que contribuyen a esta (aspiración debido a la disfunción de la deglución, alteración anatómica de la vía aérea, RGE y / o aspiración de saliva).

\section{Evidencia radiográfica de aspiración}

La radiografía simple de tórax y la tomografía computada (TC) de alta resolución son indicadores útiles de lesión pulmonar. Permiten documentar la regresión o progresión de la enfermedad a lo largo del tiempo. La TC es más sensible que la radiografía en la detección de lesiones en el parénquima pulmonar. Puede detectar bronquiectasias, atrapamiento aéreo, engrosamiento bronquial y opacidades centro-

FIGURA 2. Algoritmo para el manejo de la aspiración pulmonar crónica en el niño con enfermedad neurológica o muscular

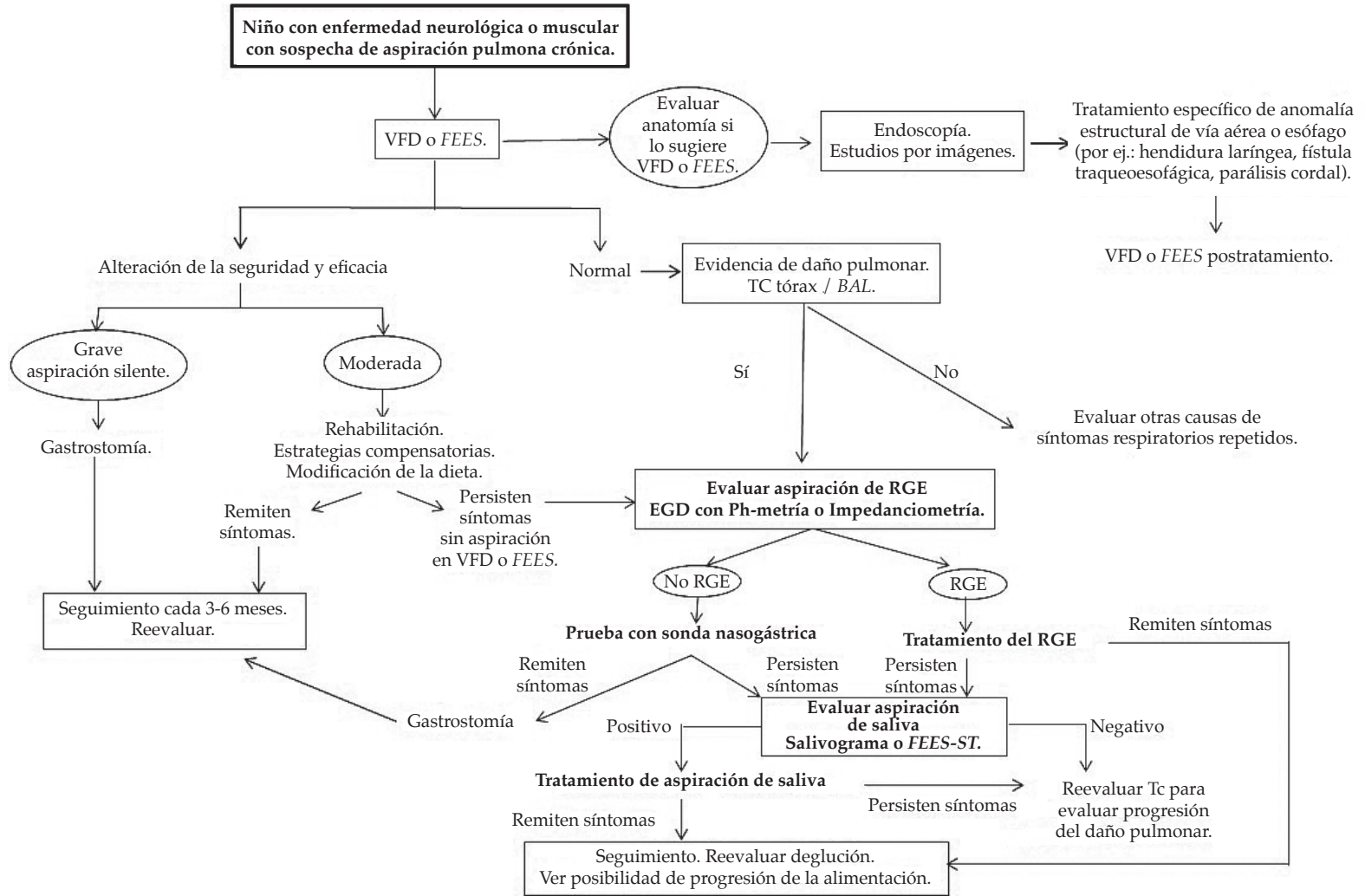

BAL: lavado broncoalveolar; EGD: esofagogastroduodenoscopía; FEES: evaluación endoscópica flexible de la deglución; FEES-ST: evaluación endoscópica flexible de la deglución con prueba sensorial; RGE: reflujo gastroesofágico;

TC: tomografía computada de tórax; VFD: videofluoroscopía de la deglución. 
lobulillares ("árbol en brote"). Los segmentos basales del lóbulo inferior y los posteriores del lóbulo superior son los más afectados. Si bien estos hallazgos no son específicos de aspiración, son comunes en los niños que se aspiran crónicamente. ${ }^{1,5}$

\section{Diagnóstico de la aspiración debido a la disfunción de la deglución y/o alteración anatómica}

Para evaluar la deglución, existen herramientas de evaluación clínica y de evaluación instrumental.

\section{EVALUACIÓN CLÍNICA DE LA DEGLUCIÓN}

La evaluación clínica de la deglución implica, además de la historia clínica y el examen físico, la evaluación, generalmente, por Fonoaudiología, de la función motora orofacial, de las estrategias de alimentación (ambiente y modo de entrega del alimento al niño) y del desarrollo de las fases de la deglución. ${ }^{2,14}$ La observación de la alimentación permite evaluar la seguridad, la eficiencia, la competencia y la confortabilidad de la deglución. La prueba de viscosidad-volumen consiste en la administración progresiva de diferentes texturas en volúmenes crecientes para detectar alteraciones de seguridad y eficacia. ${ }^{4}$ Se puede realizar con el monitoreo por oximetría de pulso; la presencia de desaturaciones es un dato indirecto de aspiración., ${ }^{4,14}$ Algunos realizan la auscultación cervical, que permite detectar patrones sonoros sugestivos de aspiración. 13,14

La evaluación clínica es fundamental, pero no es suficiente para determinar con precisión el riesgo de aspiración, debido a la alta prevalencia de aspiración silente (sin reflejo de la tos) en los niños, principalmente, en aquellos con deterioro neurológico, por lo que se debe realizar la evaluación instrumental en los pacientes con síntomas persistentes. ${ }^{9,13,14}$

Aquellos niños con trastornos neuromusculares y mal desarrollo pondoestatural, con tiempo de ingesta prolongado, que no pueden consumir los aportes calóricos diarios necesarios y sin expectativa de mejoría en menos de 6 semanas tienen indicación clínica de gastrostomía independientemente de la evaluación instrumental, la cual será de utilidad para orientar la estimulación de la deglución a seco o con alimentos.

Figura 3. Videofluoroscopia de la deglución ( $A$, B y C). Evaluación endoscópica flexible de la deglución (D, E y F)

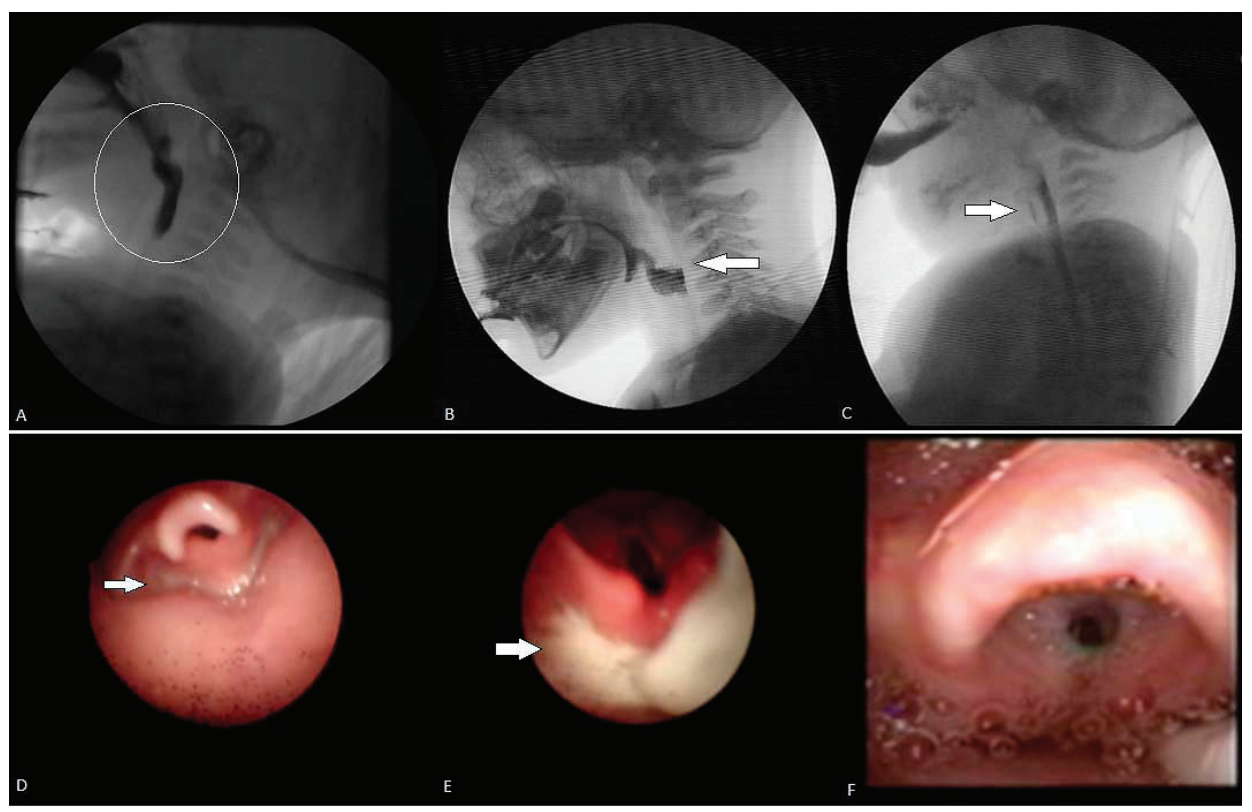

A. Fase faríngea: pasaje de contraste al esófago sin alteraciones (círculo). B. Fase faríngea: retención del contraste en vallécula e hipofaringe (flecha). C. Fase faríngea: se observa pasaje de contraste a la vía aérea (aspiración traqueal) (flecha). D. Se observa la corona laríngea de arquitectura normal y el alimento en el espacio cricofaríngeo y en los senos piriformes (flecha) antes de pasar al esófago. E. Residuos en la hipofaringe posdeglución (flecha). F. Se observan abundantes secreciones y su pasaje a la glotis (aspiración de saliva). 


\section{EVALUACIÓN INSTRUMENTAL}

La evaluación clínica inicial es seguida por la instrumental, en la que la videofluoroscopía de la deglución (VFD) y la evaluación endoscópica flexible de la deglución (Fiberoptic Endoscopic Evaluation of Swallowing; FEES, por sus siglas en inglés) son las herramientas objetivas de elección para confirmar la aspiración en la población pediátrica (Figura 3). ${ }^{114}$ Ambos son estudios complementarios; proveen de diferentes tipos de información. ${ }^{6}$ Los beneficios y las limitaciones de cada uno se resumen en la Tabla 2. ${ }^{1,25-7,710,14}$

Tanto los trastornos de la deglución faríngea (penetración y aspiración) como los trastornos esofágicos y el reflujo pueden dar lugar a neumonía recurrente. Por lo tanto, todas las fases de deglución deben ser consideradas durante la evaluación de la deglución. ${ }^{15}$

\section{Videofluoroscopía de la deglución}

La VFD es una técnica radiológica que permite evaluar en tiempo real las 3 fases de la deglución. Consiste en obtener secuencias de perfil lateral y anteroposterior (si se sospecha asimetría) mientras el paciente sentado ingiere un alimento marcado con contraste hidrosoluble o bario, en distintos volúmenes y en tres texturas distintas (semisólido, líquido y sólido). Por lo general, los padres administran al niño el alimento, y se puede utilizar el biberón o los utensilios que usan en el domicilio, así como alimentos consumidos por él habitualmente para obtener resultados más fiables.

Permite evaluar la eficacia y la seguridad de la deglución. Se puede observar anomalía en la formación del bolo, tiempo de la deglución, competencia velofaríngea, derrame posterior prematuro del bolo (antes de la deglución), residuos después de la deglución, penetración en la vía aérea (queda alimento sobre el nivel de las cuerdas vocales), aspiración traqueal con o sin tos, alteración del pasaje hacia el esófago y/o regurgitación de alimentos ingeridos.

También permite evaluar la eficacia del tratamiento. En el momento del estudio, se pueden realizar recomendaciones sobre la alimentación segura (posición óptima, consistencia y volumen de la comida, y maniobras terapéuticas). Debido a la naturaleza episódica de la aspiración, el estudio normal no descarta por completo la presencia de aspiración. . $^{1,2,5}$

\section{Evaluación endoscópica flexible de la deglución}

Se introduce un endoscopio flexible por la fosa nasal hasta visualizar la laringe y la hipofaringe, y se evalúa, en forma basal y tras la ingesta de diferentes consistencias, el proceso de la deglución. Se observa directamente la estructura y el movimiento faringolaríngeo, el manejo de las secreciones y la fase faríngea (retraso en el inicio de la deglución, presencia de residuos, reflujo a la hipofaringe, penetración y/o aspiración). También se evalúan las características indirectas de la fase oral (tiempo de deglución y posibilidad de retención del alimento en la boca). El colorante azul o verde mezclado con la comida facilita la visualización.

Puede incluir la prueba de estimulación (Fiberoptic Endoscopic Evaluation of Swallowing with Sensory Testing; FEES-ST, por sus siglas en inglés) para determinar la sensibilidad laríngea, ya sea tocar con la punta del endoscopio el repliegue aritenoepiglótico y observar la aducción cordal o aplicar una presión gradual de aire en el repliegue y documentar la presión umbral requerida para provocar el reflejo aductor laríngeo.

$\mathrm{Al}$ igual que en la VFD, se puede dar la misma comida de la casa, variar las consistencias y evaluar la eficacia de técnicas terapéuticas de deglución y de medidas compensatorias. ${ }^{1,2,5}$ No se ha evaluado en controles normales si la presencia del nasofibroscopio altera el mecanismo de la deglución. ${ }^{5}$

\section{BRONCOSCOPÍA FLEXIBLE CON LAVADO BRONCOALVEOLAR}

Permite evaluar la anatomía de la vía aérea superior e inferior, tomar muestras para el análisis citológico y microbiológico, y calcular el índice cuantitativo de macrófagos cargados con lípidos (el valor oscila entre 0 y 400). El incremento de macrófagos cargados con lípidos sugiere la aspiración directa de alimento o posterior a RGE. Este hallazgo no es específico de aspiración (se ha encontrado en la fibrosis quística y en la neumonía lipoidea, entre otras patologías), pero un valor superior a 165 es sugestivo de aspiración. ${ }^{1,5}$

\section{SERIADA ESOFAGOGASTRODUODENAL}

Evalúa, principalmente, la anatomía del tracto gastrointestinal superior. Se utiliza para evaluar RGE y aspiración, pero no es una prueba confiable por su baja sensibilidad. ${ }^{5}$ La VFD utiliza fluoroscopía continua a 30 fotogramas 
por segundo (fps), imagen enfocada en la faringe y en el esfínter esofágico superior, y distintas consistencias. En la seriada esofagogastroduodenal (SEGD), la exposición a la radiación es reducida usando una velocidad de pulso fluoroscópico menor ( $3 \mathrm{fps}$ ). La entrada de contenido a la vía aérea se subestima con menos de 30 fps. Otras limitantes de la SEGD son las siguientes: uso de fluoroscopía pulseada (que puede no capturar el episodio de aspiración), administración de contraste solo líquido, el movimiento del fluoroscopio fuera de la estructura orofaríngea para capturar el pasaje del contraste al esófago, estómago y duodeno, y la posición del paciente. ${ }^{16}$

Los niños con disfagia, a menudo, realizan ambos exámenes. En un estudio publicado, todos los pacientes con aspiración en la SEGD presentaron aspiración de líquidos en la VFD y el $53 \%$, que no tuvo aspiración en la SEGD, la presentó en la VFD. ${ }^{16}$ Incluso en el contexto de aspiración en la SEGD, la VFD es útil para definir objetivamente las alteraciones en la deglución y adoptar medidas de manejo.

\section{LARINGOTRAQUEOBRONCOSCOPÍA RÍGIDA Y/O ESOFAGOSCOPÍA}

El examen endoscópico de la vía aérea bajo anestesia general debe realizarse siempre que se sospechen anomalías anatómicas, como hendidura laríngea o fístula traqueoesofágica. También se debe considerar en el niño sin alteración neurológica con evidencia de aspiración en la VFD o FEES.

La esofagoscopía permite evaluar la anatomía $y$, si es necesario, realizar biopsias para identificar RGE o esofagitis eosinofílica.

\section{Diagnóstico de la aspiración de reflujo y de saliva}

La ph-metría y el salivograma son los estudios más utilizados para el diagnóstico de RGE y

TABla 2. Evaluación de la deglución. Beneficios y limitaciones de los diferentes exámenes complementarios

\begin{tabular}{lc}
\hline Evaluación de la deglución & Beneficios \\
\hline Videofluoroscopía de la deglución & Evalúa todas las fases \\
& de la deglución en tiempo real. \\
Evalúa varias consistencias. & Información anatómica. \\
& Las recomendaciones sobre la alimentación \\
se hacen al momento del estudio.
\end{tabular}

Limitaciones

Información limitada si el niño consume

pequeñas cantidades o no coopera.

La exposición a la radiación es

proporcional al tiempo del estudio.

No se puede realizar al lado de la cama. Evalúa un momento en el tiempo. Visión bidimensional.

Evaluación limitada de la anatomía.

Detecta menos las secreciones.

Solo evalúa la función motora.

No siempre está disponible en los centros de salud.

\begin{tabular}{|c|c|c|}
\hline $\begin{array}{l}\text { Seriada } \\
\text { esofagogastroduodenal }\end{array}$ & $\begin{array}{l}\text { Evalúa la anatomía del tracto } \\
\text { gastrointestinal superior. }\end{array}$ & $\begin{array}{l}\text { Poca sensibilidad para detectar la aspiración } \\
\text { y el reflujo gastroesofágico. }\end{array}$ \\
\hline $\begin{array}{l}\text { Evaluación endoscópica } \\
\text { flexible de la deglución }\end{array}$ & $\begin{array}{l}\text { Práctica mínimamente invasiva. } \\
\text { Evalúa la anatomía y la función de la deglución. } \\
\text { Evalúa aspectos sensitivos y motores de la deglución. } \\
\text { Visión tridimensional (más detalles anatómicos). } \\
\text { Evalúa la capacidad de protección de la vía aérea. } \\
\text { Evalúa el manejo de las secreciones orales. } \\
\text { Se puede realizar en la cama del paciente. } \\
\text { Examen portátil. } \\
\text { Más aplicable en el paciente que no coopera. } \\
\text { Puede determinar el riesgo de aspiración en el niño } \\
\text { que no se alimenta por boca. } \\
\text { Sin exposición a la radiación. } \\
\text { Se pueden visualizar múltiples degluciones } \\
\text { y se puede repetir con más frecuencia. } \\
\text { Las recomendaciones sobre la alimentación se hacen } \\
\text { al tiempo del estudio. }\end{array}$ & $\begin{array}{c}\text { Evalúa indirectamente la fase oral y } \\
\text { no evalúa la fase esofágica. } \\
\text { Se observa antes y después } \\
\text { de la deglución, no durante ella. } \\
\text { Evalúa un momento en el tiempo. } \\
\text { No está disponible ampliamente. } \\
\text { Puede no representar la situación } \\
\text { fisiológica de la deglución. } \\
\text { Requiere tolerancia al pasaje } \\
\text { del endoscopio nasal. }\end{array}$ \\
\hline $\begin{array}{l}\text { Broncoscopía flexible } \\
\text { con lavado broncoalveolar }\end{array}$ & $\begin{array}{l}\text { Evalúa la anatomía de la vía } \\
\text { aérea superior e inferior. } \\
\text { Permite tomar muestras para } \\
\text { citología y microbiología. }\end{array}$ & $\begin{array}{l}\text { No está disponible en todos los } \\
\text { centros de salud. } \\
\text { Es invasiva y requiere anestesia general. } \\
\text { Interpretación incierta del índice de } \\
\text { macrófagos cargados con lípidos. }\end{array}$ \\
\hline
\end{tabular}


de aspiración de saliva, respectivamente. Los beneficios y las limitaciones de estos estudios y otros exámenes complementarios se resumen en la Tabla 3., ${ }^{, 10,17}$

Hay niños con múltiples evaluaciones negativas para disfunción de la deglución o RGE, pero con historia clínica sugestiva de aspiración y evidencia radiológica de enfermedad pulmonar; en ellos, el diagnóstico de aspiración se hace solamente cuando se ensaya la alimentación exclusiva por sonda nasogástrica y mejoran los síntomas respiratorios. ${ }^{5}$ En los niños que continúan con síntomas de aspiración a pesar del cese de la alimentación oral y del tratamiento del

TABLA 3. Evaluación de la aspiración de reflujo gastroesofágico y de saliva. Beneficios y limitaciones de los exámenes complementarios

\begin{tabular}{lcc}
\hline $\begin{array}{l}\text { Evaluación de la aspiración } \\
\text { de reflujo gastroesofágico }\end{array}$ & Beneficios & Limitaciones \\
\hline Ph-metría esofágica & $\begin{array}{c}\text { Método de elección para el diagnóstico de RGE. } \\
\text { Datos normativos establecidos para niños. }\end{array}$ & $\begin{array}{c}\text { Es difícil establecer la relación causal } \\
\text { de RGE y aspiración. }\end{array}$ \\
& & $\begin{array}{c}\text { Es incapaz de reconocer eventos ácidos } \\
\text { superpuestos, que ocurren después de la } \\
\text { caída del ph, pero antes de normalizarse el ph. } \\
\text { No detecta RGE no ácido. }\end{array}$ \\
& Evalúa un momento en el tiempo.
\end{tabular}

\begin{tabular}{|c|c|}
\hline $\begin{array}{l}\text { Ph-impedanciometría } \\
\text { intraluminal multicanal } \\
\text { y monitorización del ph }\end{array}$ & $\begin{array}{l}\text { Detecta eventos de reflujo ácido y no ácido. } \\
\text { Detecta eventos de RGE proximal. } \\
\text { En el futuro, sería el método de elección para el } \\
\text { diagnóstico de RGE con manifestaciones supraglóticas. } \\
\text { Al detectar el RGE a nivel faríngeo, } \\
\text { puede ser capaz de predecir a aquellos niños } \\
\text { con riesgo de aspiración de RGE. } \\
\text { Permite evaluar el RGE sin interrumpir la medicación. }\end{array}$ \\
\hline
\end{tabular}

Aún no hay datos normativos para niños. Invasiva.

Es difícil de interpretar.

No disponible ampliamente.

Evalúa un momento en el tiempo. Es cara.

Permite evaluar el RGE sin interrumpir la medicación.

\begin{tabular}{lcc}
\hline $\begin{array}{l}\text { Broncoscopía flexible } \\
\text { con lavado broncoalveolar }\end{array}$ & $\begin{array}{c}\text { Permite tomar muestras para } \\
\text { citología y microbiología. } \\
\text { La detección de pepsina }\end{array}$ & $\begin{array}{c}\text { Es difícil establecer el vínculo casual entre } \\
\text { aspiración y RGE. } \\
\text { (enzima secretada por células gástricas) }\end{array}$ \\
No disponible ampliamente.
\end{tabular}

en el lavado broncoalveolar se ha propuesto

para el diagnóstico de síntomas pulmonares relacionados con RGE.

\begin{tabular}{lc}
\hline $\begin{array}{l}\text { Escintigrafía/gammagrafía } \\
\text { gastroesofágica* }\end{array}$ & $\begin{array}{c}\text { Se realiza bajo condiciones fisiológicas. } \\
\text { Baja exposición a la radiación. }\end{array}$
\end{tabular}

Poca sensibilidad.

Puede no diferenciar entre aspiración por disfunción de deglución o por RGE.

\begin{tabular}{|c|c|c|}
\hline Evaluación de la aspiración & Beneficios & Limitaciones \\
\hline Salivograma** & $\begin{array}{l}\text { Baja exposición a radiación. } \\
\text { El niño no requiere ingerir alimento. }\end{array}$ & $\begin{array}{l}\text { Sensibilidad desconocida. } \\
\text { Evalúa solo un momento. }\end{array}$ \\
\hline $\begin{array}{l}\text { Evaluación endoscópica } \\
\text { flexible de la deglución } \\
\text { con prueba sensorial }\end{array}$ & $\begin{array}{c}\text { Útil en niños que no se alimentan } \\
\text { por vía oral, pero continúan } \\
\text { con síntomas de aspiración. } \\
\text { La aspiración de las secreciones se puede } \\
\text { visualizar directamente o se puede predecir } \\
\text { la aspiración inminente por la presencia de las } \\
\text { secreciones acumuladas en la laringe y } \\
\text { la disminución de la sensibilidad laríngea. }\end{array}$ & $\begin{array}{l}\text { Evalúa un momento en el tiempo. } \\
\text { No está disponible ampliamente. } \\
\text { Requiere tolerancia al pasaje } \\
\text { de endoscopio nasal. }\end{array}$ \\
\hline Estudios con colorantes $^{* * *}$ & $\begin{array}{l}\text { Evalúa la aspiración de secreciones o de alimentos. } \\
\text { Puede ser prueba de cribado o confirmatoria. }\end{array}$ & $\begin{array}{l}\text { Solo se puede realizar en niños } \\
\text { con traqueotomía. } \\
\text { Su interpretación es incierta, } \\
\text { debido a la variabilidad de la técnica. }\end{array}$ \\
\hline
\end{tabular}

RGE: reflujo gastroesofágico.

* Consiste en mezclar tecnecio 99 en el alimento y tomar imágenes en serie para detectar la actividad del marcador en el parénquima pulmonar.

** Consiste en colocar una pequeña cantidad de radiotrazador en la boca y registrar imágenes en serie hasta que se limpie de la boca. La presencia de actividad en la tráquea y / o el bronquio indica aspiración.

*** Consiste en colocar en la lengua o en la comida un colorante, aspirar las secreciones traqueales por la cánula de traqueotomía y observar si están teñidas con el colorante o no. 
RGE, se justifica la evaluación de la aspiración de saliva.

\section{TRATAMIENTO}

El manejo de la APC requiere un enfoque multidisciplinario con la presencia del pediatra, otorrinolaringólogo, fonoaudiólogo, neumonólogo, nutricionista, gastroenterólogo, neurólogo, cirujano y psicólogo. La acción del equipo de profesionales tiene como objetivo preservar el estado nutricional y reducir el riesgo de aspiración, utilizando el método de alimentación más seguro, eficiente y mejor tolerado por el paciente. ${ }^{1,4}$

El tratamiento es individualizado, y, si es posible, se trata la causa (p. ej.: anomalía estructural de la vía aérea o del esófago).3,11 Las estrategias terapéuticas incluyen la rehabilitación postural, la modificación de la dieta (reducción del volumen de la ingesta y / o aumento de la viscosidad del bolo), los ejercicios de reforzamiento motor oral, la estimulación sensorial y / o la indicación de vías alternativas de alimentación. ${ }^{1,2,6,18}$

Cuando el paciente no puede consumir suficientes calorías por vía oral de manera segura, se indica la alimentación por sonda nasogástrica (en situaciones que no se prolonguen más de 6 semanas) o la colocación percutánea o quirúrgica de gastrostomía o yeyunostomía. ${ }^{2}$ En los niños con trastornos neurológicos o RGE sintomático, se realiza fundoplicatura en el mismo momento quirúrgico. ${ }^{5}$

Es fundamental el tratamiento del RGE. Inicialmente, se eligen terapias médicas y conservadoras: alimentos espesados, agentes procinéticos (domperidona o eritromicina) e inhibidores de la bomba de protones. En los niños con síntomas respiratorios persistentes o graves, la fundoplicatura es el tratamiento de elección. ${ }^{5}$

El tratamiento de la aspiración de saliva incluye el uso de anticolinérgicos orales (glucopirrolato), parches de escopolamina, inyección de toxina botulínica en las glándulas salivares, ligadura de los conductos submandibulares y parotídeos, y/o remoción de las glándulas submandibulares. Muchas veces, se realiza traqueotomía y, en casos graves, separación laringotraqueal. ${ }^{5}$ La higiene dental adecuada constituye un aspecto fundamental en la prevención de infecciones. Repetir el proceso de evaluación ayuda a evaluar el resultado de la intervención terapéutica. ${ }^{19}$

\section{CONCLUSIÓN}

La APC impacta significativamente en la calidad de vida del niño y su familia. El diagnóstico precoz y preciso es fundamental debido a sus potenciales complicaciones. Es muy importante identificar a aquellos niños con riesgo de aspiración, realizar las pruebas complementarias adecuadas y establecer un abordaje multidisciplinario.

Los algoritmos que se presentan aportan un ordenamiento sencillo, que se cree que será de utilidad para guiar al pediatra en el manejo de la APC en el niño. Estos algoritmos podrán ser adaptados a las condiciones de cada institución de acuerdo con los recursos disponibles, la complejidad de los pacientes y la presencia de personal con experiencia.

\section{REFERENCIAS}

1. Durvasula VS, O'Neill AC, Richter GT. Oropharyngeal dysphagiainchildren:mechanism, source, and management. Otolaryngol Clin North Am. 2014; 47(5):691-720.

2. Bacco RJL, Araya CF, Flores GE, Peña JN. Trastornos de la alimentación y deglución en niños y jóvenes portadores de parálisis cerebral: abordaje multidisciplinario. Rev Med Clin Condes. 2014; 25(2):330-42.

3. García Burriel JI. Disfagia en la infancia. An Pediatr Contin. 2014; 12(5):221-30.

4. Vaquero Sosa E, Francisco González L, Bodas Pinedo A, et al. Disfagia orofaríngea, un trastorno infravalorado en pediatría. Rev Esp Enferm Dig (Madrid). 2015; 107(2):113-5.

5. Boesch RP, Daines C, Willging JP, Kaul A, et al. Advances in the diagnosis and management of chronic pulmonary aspiration in children. Eur Respir J. 2006; 28(4):847-61.

6. Dodrill P, Gosa M. Pediatric dysphagia: Physiology, assessment, and management. Ann Nutr Metab. 2015; 66(Suppl 5):24-31.

7. Jadcherla S. Advances with neonatal aerodigestive science in the pursuit of safe swallowing in infants: Invited review. Dysphagia. 2017; 32(1):15-26.

8. LaMantia AS, Moody S, Maynard T, Karpinski B, et al. Hard to swallow: Developmental biological insights into pediatric dysphagia. Dev Biol. 2016; 409(2):329-42.

9. Arens C, Herrmann IF, Rohrbach S, Schwemmle C, et al. Position paper of the German Society of Oto-RhinoLaryngology, Head and Neck Surgery and the German Society of Phoniatrics and Pediatric Audiology - Current state of clinical and endoscopic diagnostics, evaluation, and therapy of swallowing disorders in children. GMS Curr Top Otorhinolaryngol Head Neck Surg. 2015; 14:Doc02.

10. Kim GE, Sung IY, Ko EJ, Choi KH, et al. Comparison of videofluoroscopic swallowing study and radionuclide salivagram for aspiration pneumonia in children with swallowing difficulty. Ann Rehabil Med. 2018; 42(1):52-8.

11. Svystun O, Johannsen W, Persad R, Turner JM, et al. Dysphagia in healthy children: Characteristics and management of a consecutive cohort at a tertiary centre. Int J Pediatr Otorhinolaryngol. 2017; 99:54-9.

12. Piccione J, Boesch RP. The multidisciplinary approach to pediatric aerodigestive disorders. Curr Probl Pediatr Adolesc Health Care. 2018; 48(3):66-70.

13. Zenzeri L, Quitadamo P, Tambucci R, Ummarino D, et al. Role of non-acid gastro-esophageal reflux in children with 
respiratory symptoms. Pediatr Pulmonol. 2017; 52(5):669-74.

14. Calvo I, Conway A, Henriques F, Walshe M. Diagnostic accuracy of the clinical feeding evaluation in detecting aspiration in children: a systematic review. Dev Med Child Neurol. 2016; 58(6):541-53.

15. Serel Arslan S, Demir N, Karaduman AA. Both pharyngeal and esophageal phases of swallowing are associated with recurrent pneumonia in pediatric patients. Clin Respir J. 2018; 12(2):767-71.

16. Flax-Goldenberg R, Kulkarni KS, Carson KA, Pinto JM, et al. Concordance between aspiration detected on upper gastrointestinal series and videofluoroscopic swallow study in bottle-fed children. Dysphagia. 2016; 31(4):505-10.
17. Abdallah AF, El-Desoky T, FathiK, ElkashefW, etal.Clinical utility of bronchoalveolar lavage pepsin in diagnosis of gastroesophageal reflux among wheezy infants. Can Respir J. 2016; 2016:9480843.

18. Van den Engel-Hoek L, De Groot IJ, De Swart BJ, Erasmus CE. Feeding and swallowing disorders in pediatric neuromuscular diseases: An overview. J Neuromuscul Dis. 2015; 2(4):357-69.

19. Coon E, Srivastava R, Stoddard G, Reilly S, et al. Infant videofluoroscopic swallow study testing, swallowing interventions, and future acute respiratory illness. Hosp Pediatr. 2016; 6(12):707-13.

\section{Artículos seleccionados}

Los siguientes resúmenes y comentarios de trabajos seleccionados se encuentran disponibles en la versión electrónica de este número.

Lancet. 2019 May 25;393(10186):2125-34

Levetiracetam versus fenitoína como tratamiento de segunda línea del estado epiléptico convulsivo pediátrico (estudio EcLiPSE): estudio multicéntrico aleatorizado abierto (Lyttle MD, et al. Levetiracetam versus phenytoin for second-line treatment of paediatric convulsive status epilepticus (EcLiPSE): a multicentre, open-label, randomised trial)

Comentario: Dr. Roberto Caraballo. Neurología Hospital de Pediatría “Prof. Dr. Juan P. Garrahan”

Pediatrics 2019, 143 (4) e20182012; DOI: 10.1542/ peds.2018-2012

Diferencias en las interacciones entre padres e hijos pequeños con los libros electrónicos e impresos (Munzer TG, et al. Differences in parent-toddler interactions with electronic versus print books)

Comentario: Dr. Guillermo Goldfarb. Hospital de Niños Ricardo Gutiérrez. CABA. Subcomisión de Tecnologías de Información y Comunicación - SAP

Arch Dis Child. 2019 May 11. pii: archdischild-2018-316303. [Epub ahead of print]

La adherencia al tratamiento con metformina disminuye durante las vacaciones escolares y los fines de semana en niños con diabetes tipo 1. Estudio aleatorizado controlado (Leggett $C$, et al. Adherence to metformin is reduced during school holidays and weekends in children with type 1 diabetes participating in a randomised controlled trial)

Comentario: Dra. Mabel Ferraro. Hospital Pedro de Elizalde. CABA.

Arch Dis Child. 2019 May 11. pii: archdischild-2018-316382. [Epub ahead of print]

Experiencias de los padres ante el pedido de donación de órganos y tejidos: el valor de preguntar (Darlington AS, et al. Parents' experiences of requests for organ and tissue donation: the value of asking)

Comentario: Dr. Luis Rojas. Ente Autárquico Instituto de Trasplante. Ministerio de Salud

Pediatrics 2019 JUL; 144 (1).

Patrones de pruebas de electrolitos en pacientes internados en hospitales pediátricos (Tchou $M$, et al. Patterns of electrolyte testing at children's hospitals for common inpatient diagnose)

Comentario: Dr. Gustavo M. Pereira. Cuidados Intermedios y Moderados. Hospital de Pediatría “Prof. Dr. Juan P. Garrahan"

Pediatric Pulmonology. 2018;35:1293-1302.

Farmacocinética poblacional de amikacina en pacientes con fibrosis quistica pediátrica (Caceres Guido $P$, et al.Population pharmacokinetics of amikacin in patients with pediatric cystic fibrosis)

Comentario: Dr. Fernando Rentería. Servicio de Neumonologia. Hospital Sor Ludovica. La Plata, Argentina 